\title{
Genital Mycoplasmas in Placental Infections
}

\author{
Andreas Stein, Léon Boubli, Bernard Blanc, and Didier Raoult \\ Laboratoire de Microbiologie Clinique (A.S.) and Service de Gynécologie-Obstétrique (L.B., B.B.), \\ Hôpital de la Conception, and Faculté de Médecine, Unité des Rickettsies (D.R.), Marseille, France
}

\begin{abstract}
Objective: The involvement of the genital mycoplasmas Ureaplasma urealyticum and Mycoplasma hominis in complications of pregnancy has remained controversial especially because these microorganisms are frequent colonizers of the lower genital tract. Recovery of bacteria from the placenta appears to be the sole technique to represent a true infection and not vaginal contamination. Therefore, we investigated the presence of genital mycoplasmas, aerobic and anaerobic bacteria, and fungi in human placentas and evaluated their association with morbidity and mortality of pregnancy.

Methods: We cultured placentas from 82 women with complicated pregnancies. One hundred placentas from women with uncomplicated pregnancies were evaluated as controls. When possible, placentas were examined histologically for presence of chorioamnionitis.

Results: Microorganisms were recovered from $52 \%$ of the placentas of complicated pregnancies and $U$. urealyticum was the microorganism isolated most frequently from the placenta. A significant association between positive mycoplasma culture of the placenta and complication of pregnancy was found, and chorioamnionitis was positively related to isolation of mycoplasmas.

Conclusions: These data suggest that genital mycoplasmas are able to infect the human placenta where they can cause chorioamnionitis. This infection of the placenta by genital mycoplasmas is related to preterm birth and fatal outcome of pregnancy. (c) 1994 Wiley-Liss, Inc.
\end{abstract}

KEY WORDS

Ureaplasma urealyticum, Mycoplasma hominis, morbidity of pregnancy, mortality of pregnancy

$T^{\mathrm{h}}$ he genital mycoplasmas Mycoplasma hominis and Ureaplasma urealyticum are colonizers of the female lower genital tract. ${ }^{1-3}$ These organisms have been associated with various obstetrical complications: mortality (early spontaneous abortion, late abortion, stillbirth, neonatal death) and morbidity (premature rupture of membranes, preterm birth, low birth weight). ${ }^{4}$ The involvement of $M$. hominis and $U$. urealyticum in these pathologies has been determined by isolation of these bacteria in the female lower and upper genital tracts (vagina, uterus, and amniotic fluid). ${ }^{5-9}$ However, the high rate $(10-60 \%)$ of genital colonization in the female population makes it difficult to appreciate the real pathogenic role of these 2 genital mycoplasmas in pregnancy. ${ }^{3,4,9}$ A study protocol was therefore designed to correlate isolation of these microorganisms directly from the placenta with morbidity and mortality of pregnancy. A sampling of the endometrial portion of the placenta from placentas of women with complicated pregnancies was cultured for genital mycoplasmas. A control group of placentas from uncomplicated pregnancies was similarly evaluated. All placentas were also examined for other bacterial and fungal pathogens. Placentas were examined histologically when possible and the patients' charts were reviewed to evaluate specific clinical parameters.

Address correspondence/reprint requests to Dr. Didier Raoult, Faculté de Médecine, Unité des Rickettsies, 27 Boulevard Jean Moulin, 13385 Marseille Cedex 05, France. 
TABLE I. Complicated pregnancies: maternal and fetal abnormalities indicating placenta sampling (abnormality could be associated)

\begin{tabular}{lllr}
\hline Maternal abnormalities & N & Fetal abnormalities & N \\
\hline Maternal fever & 40 & Spontaneous abortion & 12 \\
Clinical evidence of infection & 10 & Stillbirth & 6 \\
Premature rupture of membranes & 14 & Preterm birth & 13 \\
Preterm labor & 18 & Low birth weight & 4 \\
\hline
\end{tabular}

\section{SUBJECTS AND METHODS}

Women were enrolled between November 1991 and November 1992 from the Unit of Gynecology and Obstetrics of the University Hospital of Marseille, France. All women admitted to the unit were screened for eligibility. Patients were selected as follows: women with documented uterine or fetal anomalies, placenta previa, abruptio placentae, or cervical cerclage were excluded from the study and women presenting with at least one maternal or fetal abnormality listed in Table 1 were included in the study. The placentas of these subjects were submitted to the laboratory for microbiological examination and culture. Placentas from women with uncomplicated pregnancies and none of the fetal or maternal abnormalities listed in Table 1 were evaluated as controls. Samples of the maternal surface of the placenta were obtained with a sterile surgical blade by slicing a $1.0 \times 1.0 \times 1.0 \mathrm{~cm}$ portion of the depth of the placenta. The samples were ground with a sterile tissue grinder and examined following Gram staining. Aerobic and anaerobic cultures for bacteria and fungi were made. Mycoplasmas were cultured in both liquid and agar media. Bacteria and fungi were identified according to the technique of Balows et al. ${ }^{10}$ For culture of mycoplasmas, the placental tissue suspension was transferred onto a Mycofast identification strip (Groupe Stago, International Mycoplasma, Signes, France) and selective A-7 agar medium (Groupe Stago, International Mycoplasma) following the manufacturers' instructions. ${ }^{11,12}$ Both the Mycofast strip and A-7 plates were incubated for 7 days at $37^{\circ} \mathrm{C}$ in a $\mathrm{CO}_{2}$ atmosphere and were examined daily for the presence of $U$. urealyticum and/or $M$. hominis. Culture confirmation and numeration of mycoplasma were based on the detection of the color changes in the Mycofast identification strip and on the colonial morphology in A-7 agar. All placentas were cul- tured on the same day of delivery without knowledge of the subject's status. When the attending physician requested it, the placenta was examined histologically without knowledge of the results of cultures or the status of the subject. The reasons for histopathological examination of the placenta included stillbirth, neonatal death, and complications of pregnancy, labor, or delivery. Histological chorioamnionitis was defined as the demonstration of inflammatory cells, predominantly neutrophils, in the chorion and amnion. ${ }^{13}$

The medical records of all women were reviewed. Gestational age was estimated from the date of the mother's last menstruation, fundal height, and ultrasonography. Prematurity was defined as a gestational age of $<35$ weeks. Fatal outcome of pregnancy was defined as mortality due to early or late abortion, death in utero, or stillbirth.

The distribution of the culture results of the microorganisms among the study groups was examined. A $\chi^{2}$ test was used to evaluate the equality of proportions of positive cultures and presence of chorioamnionitis in the study groups and to correlate ratios of positive culture, chorioamnionitis, prematurity, and fatal outcome of pregnancy.

\section{RESULTS}

In all, 82 placentas were obtained from women with complicated pregnancies and 100 placentas from women with none of the fetal or maternal abnormalities listed in Table 1 were used as controls.

The recovery of $U$. urealyticum, $M$. hominis, and other bacteria from the placenta in both groups is shown in Table 2. Microorganisms were recovered from $43(52 \%)$ of the 82 placentas from complicated pregnancies. Twenty-two placentas $(27 \%)$ contained genital mycoplasmas and 21 placentas (25\%) other bacteria. Only 4 placentas (4\%) of the control group grew microorganisms. The variation of the isolation rate of microorganisms among the study group and the control group was statistically significant $\left(P<10^{-8}\right)$. In the study group, $U$. urealyticum was the microorganism isolated most frequently from the placenta (24\%). These data show a significant association between positive mycoplasma cultures of the placenta and complications of pregnancy $\left(P<10^{-6}\right)$.

The results of the histopathological examination of the placenta are shown in Table 3. In all, 61 of 
TABLE 2. Microorganisms recovered from placental cultures

\begin{tabular}{|c|c|c|c|c|c|}
\hline & \multicolumn{2}{|c|}{$\begin{array}{c}\begin{array}{c}\text { Study } \\
\text { group } \\
(\mathrm{N}=82) \\
\end{array} \\
\end{array}$} & \multicolumn{2}{|c|}{$\begin{array}{c}\begin{array}{c}\text { Control } \\
\text { group } \\
(N=100) \\
\end{array} \\
\end{array}$} & \multirow[b]{2}{*}{$P$} \\
\hline & $N$ & $\%$ & $N$ & $\%$ & \\
\hline \multicolumn{6}{|l|}{ Genital mycoplasmas } \\
\hline Ureaplasma urealyticum ${ }^{\mathrm{a}}$ & 20 & 24 & 1 & 1 & $<10^{-6}$ \\
\hline Mycoplasma hominis & 2 & 2.4 & & & \\
\hline \multicolumn{6}{|l|}{ Facultative bacteria } \\
\hline Group B Streptococcus & 3 & 3.7 & & & \\
\hline Viridans streptococci & 3 & 3.7 & & & \\
\hline Enterococcus & 3 & 3.7 & & & \\
\hline Escherichia coli & 5 & 6 & 1 & 1 & \\
\hline Staphylococcus aureus & 1 & 1.2 & & & $<10^{-5}$ \\
\hline \multicolumn{6}{|l|}{ Anaerobic bacteria } \\
\hline Peptostreptococcus & 4 & 4.9 & & & \\
\hline Clostridium & 1 & 1.2 & 2 & 2 & \\
\hline \multicolumn{6}{|l|}{ Yeast } \\
\hline Candida albicans & $\mathrm{I}$ & 1.2 & & & \\
\hline Total microorganisms & 43 & 52 & 4 & 4 & $<10^{-8}$ \\
\hline Sterile & 39 & 48 & 96 & 96 & \\
\hline
\end{tabular}

aU. urealyticum was associated in I placenta of the study group with group $B$ streptococci and in another placenta with $E$. coli.

TABLE 3. Association of bacterial isolates from the placenta with histological chorioamnionitis

\begin{tabular}{lcc}
\hline & \multicolumn{2}{c}{$\begin{array}{c}\text { Chorioamnionitis } \\
\text { (placentas/placentas examined) }\end{array}$} \\
\cline { 2 - 3 } & $\mathrm{N}$ & $\%$ \\
\hline Genital mycoplasmas & & \\
Ureaplasma urealyticum & $11 / 16$ & 69 \\
Mycoplasma hominis & - & - \\
Facultative bacteria & & \\
Group B Streptococcus & $2 / 2$ & 100 \\
Viridans streptococci & $3 / 3$ & 100 \\
Enterococcus & $2 / 3$ & 66 \\
Escherichia coli & $4 / 5$ & 80 \\
Staphylococcus aureus & $1 / 1$ & 100 \\
Anaerobic bacteria & & 50 \\
Peptostreptococcus & $1 / 2$ & 100 \\
$\quad$ Clostridium & $1 / 1$ & 76 \\
Total microorganisms & $25 / 33$ & 32 \\
Sterile & $9 / 28$ & \\
\hline
\end{tabular}

the 82 placentas from complicated pregnancies were examined histopathologically. Of these, 34 placentas $(56 \%)$ showed histopathological chorioamnionitis. Chorioamnionitis was seen in $69 \%$ of the 16 placentas from which mycoplasmas were isolated;
$32 \%$ of the sterile placentas of the study group showed inflammation. This difference in occurrence of chorioamnionitis was significant $(P<0.002)$. When comparing the sterile placentas, we noted that recovery of genital mycoplasmas or other bacteria of the placenta was significantly related to preterm birth and mortality of pregnancy (Table 4). In the group of placentas growing genital mycoplasmas, $27 \%$ were associated with preterm birth $(P<0.03)$ and $45 \%$ to fatal outcome of the pregnancy $\left(P<10^{-4}\right)$. In the group of placentas infected with bacteria other than genital mycoplasmas, $24 \%$ were associated with preterm birth $(P<0.05)$ and $33 \%$ with fatal outcome of pregnancy $(P<0.002)$. Recovery of genital mycoplasmas and other bacteria was significantly related to preterm birth and mortality of pregnancy (Table 4).

\section{DISCUSSION}

The involvement of the genital mycoplasmas $U$. urealyticum and $M$. hominis in mortality and morbidity of pregnancy was initially suggested by isolation of these bacteria in the female lower and upper genital tracts (vagina, uterus, amniotic fluid, and endometrium $)^{5-9}$ or by serological diagnosis. ${ }^{14,15}$ However, the role of genital mycoplasma infection in complications of pregnancy has remained controversial especially because these microorganisms frequently colonize the lower genital tract of pregnant women who are not ill and who give birth to normal, healthy infants. In fact, the high prevalence of genital colonization in the female population, which ranges from 10 to $60 \%$, makes it difficult to accept that in some patients mycoplasmas are pathogenic, while in other there is no evidence of infection. ${ }^{2} \mathrm{~A}$ recent review on the role of $U$. urealyticum in premature birth demonstrated that $U$. urealyticum in the lower genital tract is not directly associated with preterm birth, as preterm birth is related to a variety of risk factors for prematurity (black race, young maternal age, low educational level, low income, smoking during pregnancy, history of marijuana and/or cocaine use, and separate, single marital status). The authors conclude that these factors may be interdependent and that no consistent cause-andeffect relationship exists between the presence of $U$. urealyticum in the lower genital tract of the mother and prematurity. ${ }^{9}$ This opinion is supported by the findings of 2 studies in which erythromycin was 
TABLE 4. Relationship of preterm birth and fatal outcome (abortion, intrauterine decease, stillbirth) of the pregnancy to bacterial infection of the placenta

\begin{tabular}{|c|c|c|c|c|c|c|c|c|}
\hline & \multicolumn{2}{|c|}{$\begin{array}{l}\text { Placentas growing } \\
\text { genital mycoplasmas } \\
\qquad(\mathrm{N}=22)\end{array}$} & \multicolumn{2}{|c|}{$\begin{array}{l}\text { Placentas growing bacteria } \\
\text { other than mycoplasmas } \\
\qquad(\mathrm{N}=2 \mathrm{I})\end{array}$} & \multicolumn{2}{|c|}{$\begin{array}{l}\text { Sterile placentas } \\
\quad(\mathrm{N}=39)\end{array}$} & \multicolumn{2}{|c|}{$\begin{array}{c}\text { Total } \\
(\mathrm{N}=\mathbf{8 2})\end{array}$} \\
\hline & $\mathbf{N}$ & $\%$ & $N$ & $\%$ & $\mathbf{N}$ & $\%$ & $\mathrm{~N}$ & $\%$ \\
\hline Preterm birth & 6 & 27 & 5 & 24 & 2 & 5 & 13 & 16 \\
\hline Fatal outcome & 10 & 45 & 7 & 33 & 1 & 2.5 & 18 & 22 \\
\hline
\end{tabular}

used to treat women with genital colonization by mycoplasmas. In these 2 studies, it was demonstrated that erythromycin treatment in women with $U$. urealyticum in the lower genital tract had virtually no impact upon low birth weight or prematurity. ${ }^{16,17}$ As there are case reports implicating $U$. urealyticum in clinical amnionitis, investigators tried to correlate the presence of mycoplasmas in the amniotic fluid with the morbidity of pregnancy. ${ }^{7,18}$ However, mycoplasmas are present in $50 \%$ of amniotic fluid samples from both women with intraamniotic infection and asymptomatic control women and $U$. urealyticum does not appear to be associated with clinically evident intra-amniotic mycoplasmal infection. ${ }^{19}$

In fact, the recovery of bacteria from the placenta appears to be the sole technique to represent a true infection and not vaginal contamination, ${ }^{9}$ as bacteria from the typical vaginal flora are almost never recovered from placental specimens. Furthermore, the relationship between infection of the placenta and prematurity has been shown to be independent of the duration of labor, the presence of ruptured membranes, or the duration of ruptured membranes. ${ }^{20}$ These findings suggest that infection occurs before labor and is not a result of prolonged labor or rupture of membranes. So far, only 7 studies have examined the relationship between colonization of the placenta and outcome of pregnancy. Naessens et al. ${ }^{21}$ cultured placentas for $U$. urealyticum but not $M$. hominis from 253 women and found no significant difference in the birth weight of infants whose placentas were colonized. Two other studies showed that the presence of $U$. urealyticum in the placenta was not related to low birth weight or prematurity. ${ }^{22,23}$ Embree et al. ${ }^{24}$ cultured placentas from a group of 446 "high-risk" pregnancies and 108 unselected deliveries of nor- mal full-term infants and found an association of the isolation of $U$. urealyticum with prematurity, lower birth weight, and intrauterine growth retardation. Kundsin et al. ${ }^{25}$ confirmed those data in a prospective study by isolating mycoplasmas more frequently from the placentas of infants who died in the perinatal period and from the placentas of those neonates admitted to the intensive case unit than from matched controls. Hillier et al. ${ }^{20}$ related both the isolation of $U$. urealyticum alone or with other bacteria and the isolation of bacteria without $U$. urealyticum to premature delivery. In these latter 3 studies, $U$. urealyticum was isolated from the chorioamnion of 24-47\% of patients who delivered low birth weight, premature infants and from 9-19\% of patients who delivered at term. The frequency of isolation of $U$. urealyticum from the chorioamnion was significantly higher in those who delivered prematurely compared with those who delivered at term in these 3 studies.

As for the role of mycoplasmas in the fatal outcome of pregnancy, the 1st reports on the isolation of genital mycoplasmas from stillbirth were based on individual cases in which these organisms were isolated from fetal lungs, brain, heart, and viscera $^{26-30}$ and on 2 studies in which $U$. urealyticum was isolated more frequently from the products of early abortions and mid-trimester fetal losses than from products of induced abortions. ${ }^{31,32}$ Embree et al. ${ }^{24}$ isolated $U$. urealyticum more frequently from placentas of aborted fetuses than from controls. In another study of 33 perinatal death and 31 random cases of normal term deliveries, Quinn et al. ${ }^{33}$ isolated genital mycoplasmas significantly more often from cases in which death of the fetus could not be attributed to a known anatomic or morphologic cause than from controls. Unfortunately, these authors combined their culture data 
with serological results and cases were considered positive if they were serologically positive even though they were culture negative.

Histological chorioamnionitis has consistently been related to morbidity and mortality of pregnancy. What causes chorioamnionitis is still a controversial subject because of the diversity of microorganisms isolated, often of low virulence, and the concept that non-specific placental inflammation may occur. ${ }^{34}$ As related to the cause of perinatal death, no morphologic cause could be documented at autopsy of the fetus in nearly $2 / 3$ of cases and it was shown that chorioamnionitis occurs in more than $50 \%$ of these stillbirths and early neonatal deaths. ${ }^{33,35}$ Moreover, the rates of histological chorioamnionitis are 2 - to 5 -fold higher in patients who deliver preterm than in those who deliver at term $^{20}$ and a direct relationship exists between the degrees of prematurity and the prevalence of histological chorioamnionitis. ${ }^{13}$ It was shown that bacteria are recovered from the placenta of $70 \%$ of patients with histological chorioamnionitis but from only $15-45 \%$ of patients without histological chorioamnionitis. ${ }^{36} U$. urealyticum was isolated from the chorioamnion of $38-66 \%$ of patients with histological chorioamnionitis and from $13-17 \%$ of patients without histological chorioamnionitis. All of these studies which associated microbiological and histological examination of placentas revealed an increased prevalence of chorioamnionitis, spontaneous abortion, stillbirth, neonatal death, and perinatal morbidity among women with mycoplasmas isolated from the placenta compared with cases which were culture negative. ${ }^{20,24,25,35}$ These studies suggest that $U$. urealyticum associated with chorioamnionitis may be a major factor in placental infections leading to perinatal morbidity and mortality. The aim of our study was to determine if placental infections and particularly infections with mycoplasmas represent 1 cause of preterm birth and/or fatal outcome of pregnancy. We studied the relationship between morbidity (prematurity) and mortality of pregnancy with both microbiologic and histologic findings of the placenta. A recent review of the 7 reports concerning isolation of mycoplasmas in the placenta suggests that the presence of $U$. urealyticum in the chorioamnion is only weakly associated with prematurity but strongly associated with histological chorioamnionitis. ${ }^{9}$ Our results demonstrate an association between histological chorioamnionitis and the recovery of microorganisms from the placenta. In all, we found that in the group of complicated pregnancies $76 \%$ of the placentas growing mycoplasmas or other bacteria showed histological inflammation compared with $32 \%$ of the sterile placentas. $U$. urealyticum was the microorganism most frequently isolated from the placentas of complicated pregnancies. A lack of vaginal contamination was suggested by the absence of Lactobacillus isolated from the placental cultures. The presence of $U$. urealyticum in the chorioamnion appears to cause histological chorioamnionitis in the same manner as the well-known placental pathogens Escherichia coli and group B Streptococcus. Furthermore, chorioamnionitis and isolation of specific microorganisms from the placenta are associated with a significant incidence of prematurity and fatal outcome of pregnancy.

As for prematurity, pregnancies with placentas harboring genital mycoplasmas or other bacteria had significantly higher preterm birth rates $(27$ and $24 \%$, respectively) than complicated pregnancies with sterile placentas (5\%). As for fatal outcome of pregnancy, previous studies showed an increased incidence of chorioamnionitis and a higher isolation rate of $U$. urealyticum in perinatal deaths compared with controls, especially among those with no anatomic cause of death. In our study, chorioamnionitis was observed in $68 \%$ of pregnancies with fatal outcome. We observed a higher rate of fatal outcome in the group of pregnancies with placentas growing genital mycoplasmas (45\%) than in the group growing bacteria other than mycoplasmas $(33 \%)$ or in the group of sterile placentas $(2.5 \%)$. These results are consistent with the report of recovery of $U$. urealyticum from discolored amniotic fluid among patients undergoing amniocentesis at 16-20 weeks gestation for genetic evaluation. In this study, patients with $U$. urealyticum in the discolored amniotic fluid delivered prematurely with evidence of severe histological chorioamnionitis and fetal $U$. urealyticum infection. ${ }^{8}$ Furthermore, in a bovine model, ureaplasmas that were experimentally inoculated into the amniotic flud of pregnant heifers were shown to induce abortion and premature delivery associated with severe placentitis. ${ }^{37,38}$

In conclusion, our findings suggest that $U$. ure- 
alyticum is emerging as an important placental pathogen. The colonization of the placenta with this microorganism, frequently associated with chorioamnionitis, constitutes a major factor in intrauterine infections associated with morbidity and mortality of pregnancy. Unfortunately, no clinically useful methods to diagnose placental infections before delivery are available. On the basis of our results, we recommend that histological and microbiological examination of the placenta be done in all cases of complicated pregnancies. Only in this manner can we better understand the pathogenesis of placental infections and develop diagnostic and therapeutic tools to improve morbidity and mortality of these pregnancies.

\section{REFERENCES}

1. Taylor-Robinson D, McCormack WM: The genital mycoplasmas. N Engl J Med 302:1063-1067, 1980.

2. Cassell GH, Cole BC: Mycoplasmas as agents of human disease. N Engl J Med 304:80, 1981.

3. Taylor-Robinson D: Ureaplasma urealyticum (T-strain mycoplasma) and Mycoplasma hominis. In Mandell GL, Douglas RG, Bennett JE (eds): Principles and Practice of Infectious Diseases. New York: Churchill Livingstone, pp 1458-1463, 1990.

4. Cassell GH, Davis JK, Waites KB, Rudd PT, Talkington $\mathrm{D}$, Crouse D, Horowitz SA: Pathogenesis and significance of urogenital mycoplasmal infections. Adv Exp Med Biol 224:93-115, 1987.

5. Romero R, Sirtori M, Oyarzun E: Infection and labor. V. Prevalence, microbiology, and clinical significance of intraamniotic infection in women with preterm labor and intact membranes. Obstet Gynecol 161:817-824, 1989.

6. Romero R, Mazor M, Oyarzun E, Sirtori M, Wu YK, Hobbins JC: Is genital colonization with Mycoplasma hominis or Ureaplasma urealyticum associated with prematurity/low birth weight? Obstet Gynecol 73:532536, 1989.

7. Gravett MG, Hummel D, Eschenbach DA, Holmes KK: Preterm labor associated with subclinical amniotic fluid infection and with bacterial vaginosis. Obstet Gynecol 67:229-237, 1986.

8. Cassell GH, Davis RO, Waites KB: Isolation of Mycoplasma hominis and Ureaplasma urealyticum from amniotic fluid at 16-20 weeks of gestation: Potential effect on outcome of pregnancy. Sex Transm Dis 10:294, 1983.

9. Eschenbach DA: Ureaplasma urealyticum and premature birth. Clin Infect Dis 17:100-106, 1993.

10. Balows A, Hausler WJ, Hermann KL, Isenberg HD, Shadomy HJ: Manual of Clinical Microbiology. 5th ed. Washington, DC: American Society of Microbiology, 1991.

11. Kundsin RB, Parreno A, Poulin S: Significance of appropriate techniques and media for isolation and identifica- tion of Ureaplasma urealyticum from clinical specimens. J Clin Microbiol 8:445-453, 1978.

12. Shepard MC, Lunceford CD: Differential agar medium (A7) for identification of Ureaplasma urealyticum (human T mycoplasmas) in primary culture of clinical material. J Clin Microbiol 3:613-625, 1976.

13. Russel P: Inflammatory lesions of the human placenta. Clinical significance of acute chorioamnionitis. Am J Diagn Gynecol Obstet 1:127-137, 1979.

14. Quinn PA, Shewchuk AB, Shubern J: Serologic evidence of Ureaplasma urealyticum infection in women with spontaneous pregnancy loss. Am J Obstet Gynecol 145:245$250,1983$.

15. Gibbs RS, Cassell GH, Davis JK: Further studies on genital mycoplasmas in intra-amniotic infection: Blood cultures and serologic response. Am J Obstet Gynecol 154:717-726, 1986.

16. McCormack WM, Rosner BL, Lee YH, Munoz A, Charles D, Kass EH: Effect on birth weight of erythromycin treatment of pregnant women. Obstet Gynecol 69: 202-207, 1987.

17. Eschenbach DA, Nugent RP, Rao AV: A randomized placebo controlled trial of erythromycin for the treatment of Ureaplasma urealyticum to prevent premature delivery. Am J Obstet Gynecol 64:734-742, 1991.

18. Cassell GH, Waites KB, Gibbs RS, Davis JK: Role of Ureaplasma urealyticum in amnionitis. Pediatr Infect Dis 5S:341-344, 1986.

19. Blanco JD, Gibbs RS, Malherbe H: A controlled study of genital mycoplasmas in amniotic fluid from patients with intra-amniotic infection. J Infect Dis 147:650-653, 1983.

20. Hillier SL, Martius J, Krohn M, Kiviat N, Holmes KK, Eschenbach DA: A case control study of chorioamnionic infection and histologic chorioamnionitis in prematurity. N Engl J Med 319:972-978, 1988.

21. Naessens A, Foulon W, Breynaert J, Lauwers S: Postpartum bacteremia and placental colonization with genital mycoplasmas and pregnancy outcome. Am J Obstet Gynecol 160:647-650, 1989

22. Hillier S, Krohn MA, Kiviat NB, Watts DH, Eschenbach DA: Microbiologic causes and neonatal outcomes associated with chorioamnion infection. Am J Obstet Gynecol 165:955-961, 1991.

23. Zlatnik FJ, Gelhaus TM, Benda JA, Koontz FP, Burmeister LF: Histologic chorioamnionitis, microbial infection and prematurity. Obstet Gynecol 76:355-359, 1990.

24. Embree JE, Krause VW, Embil JA, McDonald S: Placental infection with Mycoplasma hominis and Ureaplasma urealyticum, clinical correlation. Obstet Gynecol 56:475$481,1980$.

25. Kundsin RB, Driscoll SG, Monson RR, Yeh C, Biano SA, Cochran WD: Association of Ureaplasma urealyticum in the placenta with perinatal morbidity and mortality. N Engl J Med 310:941-945, 1984.

26. Quinn PA, Gilan JE, Markstad T: Intrauterine infection with Ureaplasma urealyticum as a cause of fatal neonatal pneumonia. Pediatr Infect Dis 4:538, 1985. 
27. Brunnell PA, Dische MR, Walker M: Mycoplasma amnionitis and respiratory distress syndrome. JAMA 207: 2097, 1969.

28. Romano N, Romano F, Carollo F: T-strains of mycoplasmas in broncopneumonic lungs of an aborted fetus. $\mathrm{N}$ Engl J Med 285:950, 1971.

29. Pease P, Rogers KB, Cole BC: A cytopathogenic strain of Mycoplasma hominis type I isolated from the lung of a stillborn infant. J Pathol Bacteriol 94:460, 1967.

30. Tafari N, Ross S, Naeye RL: Mycoplasma T strains and perinatal death. Lancet 1:108, 1976.

31. Sompolinsky D, Solomon F, Eikina L: Infections with mycoplasma and bacteria in induced mid-trimester abortion and fetal loss. Am J Obstet Gynecol 121:610, 1975.

32. Caspi E, Solomon F, Sompolinsky D: Early abortion and Mycoplasma infection. Isr J Med Sci 8:122, 1972.

33. Quinn PA, Butany J, Chipman M: A prospective study of microbial infection in stillbirth and early neonatal death. Am J Obstet Gynecol 151:238-249, 1985.
34. Pankuch GA, Appelbaum PC, Lornz RP, Botti JJ, Schachter L, Nave RL: Placental microbiology and histology and the pathogenesis of chorioamnionitis. Obstet Gynecol 64:802-806, 1984.

35. Quinn PA, Butany J, Taylor J, Hannah W: Chorioamnionitis: Its association with pregnancy outcome and microbial infection. Am J Obstet Gynecol 156:379-387, 1987.

36. Gibbs RS, Romero R, Hillier SL, Eschenbach DA, Sweet RL: A review of premature birth and subclinical infection. Am J Obstet Gynecol 166:1515-1518, 1992.

37. Miller RB, Ruhnke RL, Doig BF: The effects of Ureaplasma diversum inoculated into the amniotic cavity in cows. Theriogenology 20:367, 1983.

38. Ruhnke RL, Palmer NC, Doig PA: Bovine abortion and neonatal death associated with Ureaplasma diversum. Theriogenology 21:295, 1984 . 


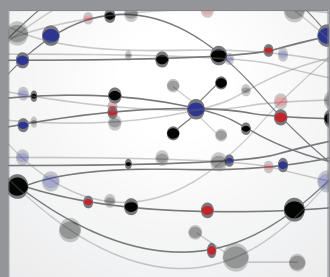

The Scientific World Journal
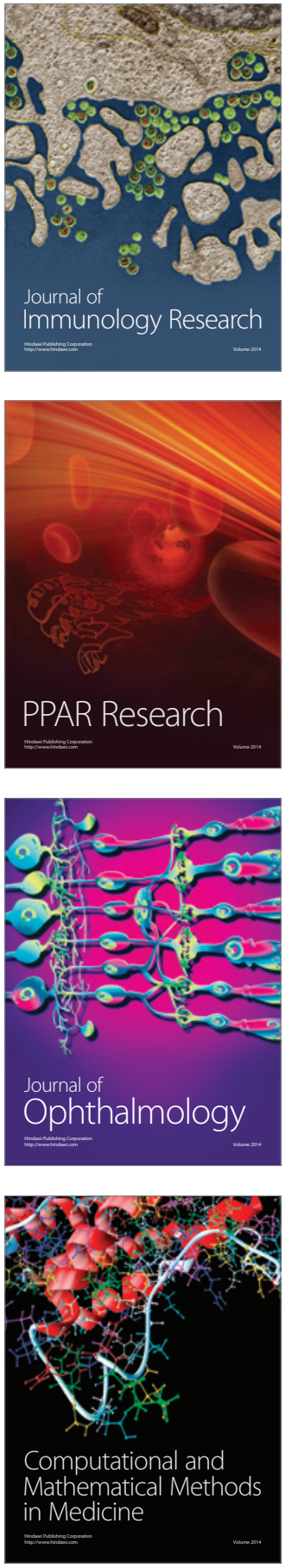

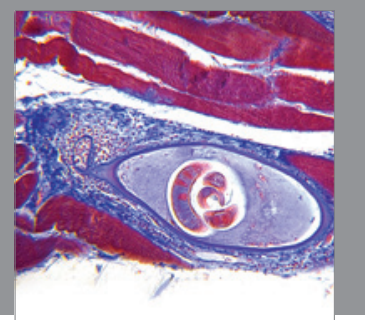

Gastroenterology

Research and Practice
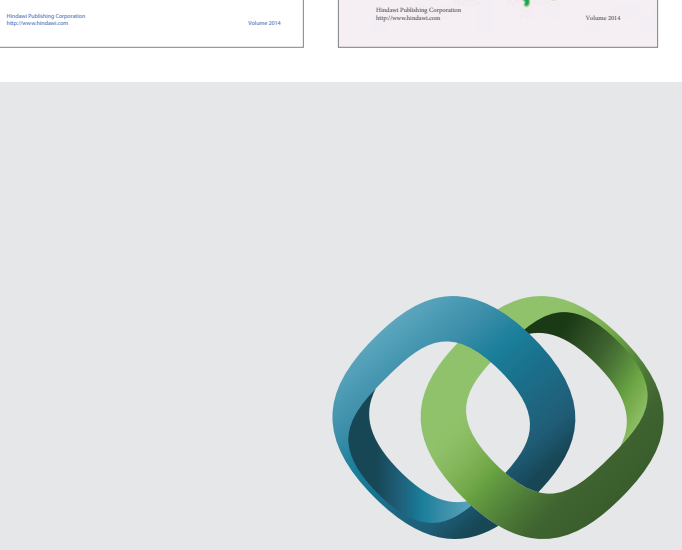

\section{Hindawi}

Submit your manuscripts at

http://www.hindawi.com
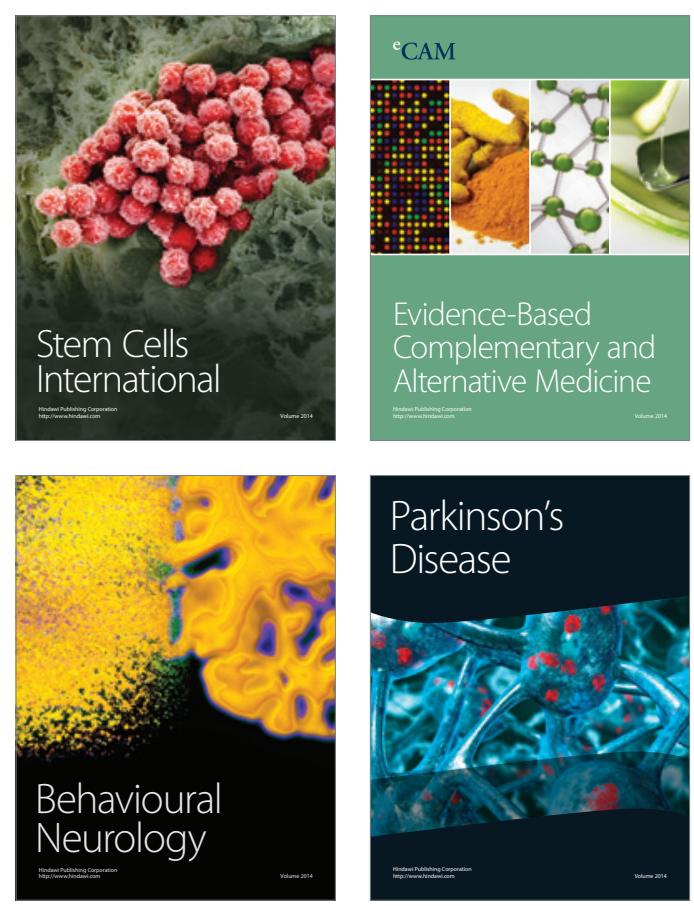

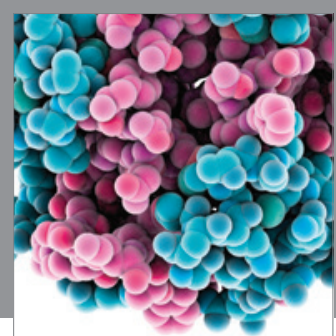

Journal of
Diabetes Research

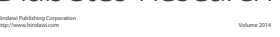

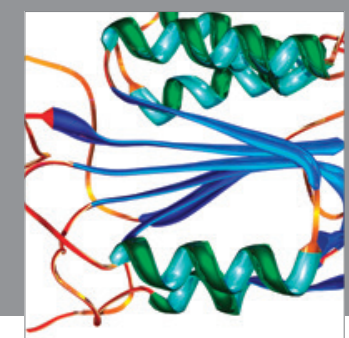

Disease Markers
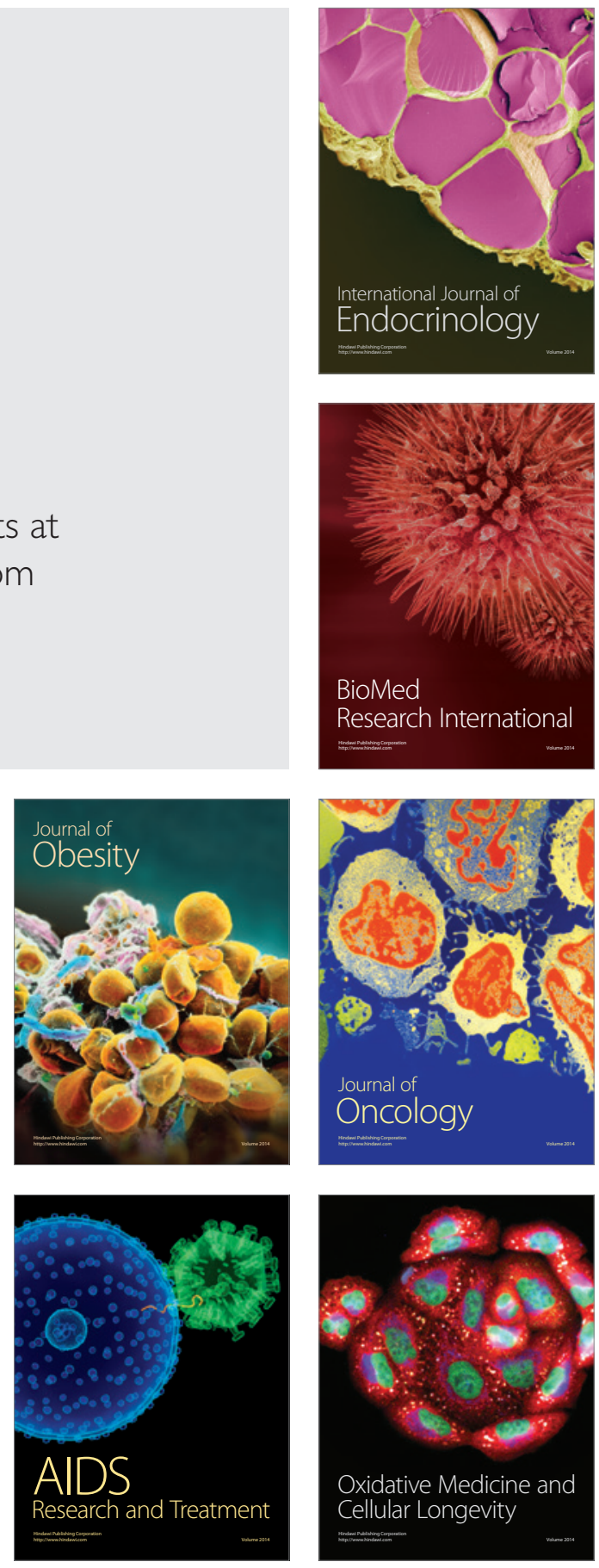